\title{
MIGRAÇÃO DE PARAFUSO EM ARTROPLASTIA TOTAL DO JOELHO: RELATO CLÍNICO
}

\author{
SCREW MIGRATION IN TOTAL KNEE ARTHROPLASTY. CASE REPORT
}

Fernando Fonseca' ${ }^{1}$, José Tomé2 ${ }^{2}$, Manuel Barreto ${ }^{3}$

\section{RESUMO}

As complicações das artroplastias totais do joelho devidas ao material implantado são raras, exceto o desgaste do polietileno. O relato de migração de parafusos dentro da articulação do joelho é muito raro. Os autores relatam a migração intra-articular de um parafuso de segurança do polietileno numa artroplastia total do joelho com sacrifício do ligamento cruzado posterior (ATJ tipo Performance; Biomet, Warsaw, IN) que obrigou a nova cirurgia para remoção do parafuso, substituição do componente de polietileno e aplicação de novo parafuso de fixação.

Descritores - Artroplastia do joelho; Articulação do joelho; Revisão; Parafusos ósseos; Migração de corpo estranho

\section{ABSTRACT}

Complications in total knee arthroplasty directly related to the implanted material are rare, with the exception of polyethylene wear. Descriptions of screw migration into the joint cavity are very rare. The authors report an intra-articular migration of a polyethylene safety screw in a total knee arthroplasty, with sacrifice of the posterior cruciate ligament (ATJ Performance type; Biomet, Warsaw, IN) requiring a new surgery to remove the screw, replace the polyethylene insert and insert a new fixation screw.

Keywords - Arthroplasty, Replacement, Knee; Knee joint; Review; Bone screws; Foreign-Body Migration

\section{INTRODUÇÃO}

Os relatos de complicações de artroplastias totais do joelho relacionadas com o material (ATJ) são raros, excepto o desgaste do polietileno ${ }^{(1-5)}$.

A descrição de afrouxamento e migração de parafusos no interior do joelho é também muito escassa na literatura ${ }^{(6,7)}$. Os autores descrevem um caso de migração de parafuso do componente tibial de uma ATJ (Performance Knee; Biomet, Warsaw, IN) que obrigou a nova cirurgia para substituição do polietileno e sua fixação com parafuso.

\section{RELATO DE CASO}

Em Fevereiro de 2005 uma paciente de 67 anos de idade foi operada ao joelho esquerdo por osteonecrose do côndilo femoral medial, tendo-lhe sido implantada uma ATJ cimentada com sacrifício do ligamento cruzado posterior (Performance; Biomet, Warsaw, IN). Como tinha grande desgaste do planalto tibial, foram utilizadas haste femoral, haste tibial e step tibial.

O pós-operatório decorreu sem incidentes, ficando a paciente com um arco de movimento final de $0^{\circ}$ a $110^{\circ}$. As radiografias mostravam a prótese bem implantada, bem alinhada e sem radiolucência.

Dois anos depois, Fevereiro de 2007, a paciente refere a sensação de instabilidade no joelho operado, recorrendo ao ambulatório do nosso hospital. Na entrada não referia dor, a marcha era normal sem claudicação, mas com auxílio de uma canadiana. Apresentava um pequeno derrame no joelho operado. No plano frontal tinha uma ligeira abertura em valgo e em varo com o joelho em extensão. Referia a sensação de um corpo estranho intra-articular que se movia.

1 - Professor Auxiliar. Chefe do Setor Joelho dos Hospitais da Universidade de Coimbra, Portugal.

2 - Especialista. Membro do Setor Joelho dos Hospitais da Universidade de Coimbra, Portugal.

3 - Especialista. Membro do Corpo Clínico da Casa de Saúde Coimbra, Portugal.

Trabalho realizado no Setor de Joelho do Serviço de Ortopedia dos Hospitais da Universidade de Coimbra, Portugal.

Correspondência: Serviço de Ortopedia A, Hospitais da Universidade de Coimbra.

Praceta Mota Pinto - 3000 - Coimbra, Portugal. E-mail: pereirafonseca@clix.pt 
O exame radiográfico (Figura 1) mostrou a presença do parafuso de fixação do polietileno migrado no interior da cavidade articular. Em face do observado decidiu-se proceder à cirurgia para revisão de ATJ por receio de dano nas superfícies articulares, nomeadamente a femoral.

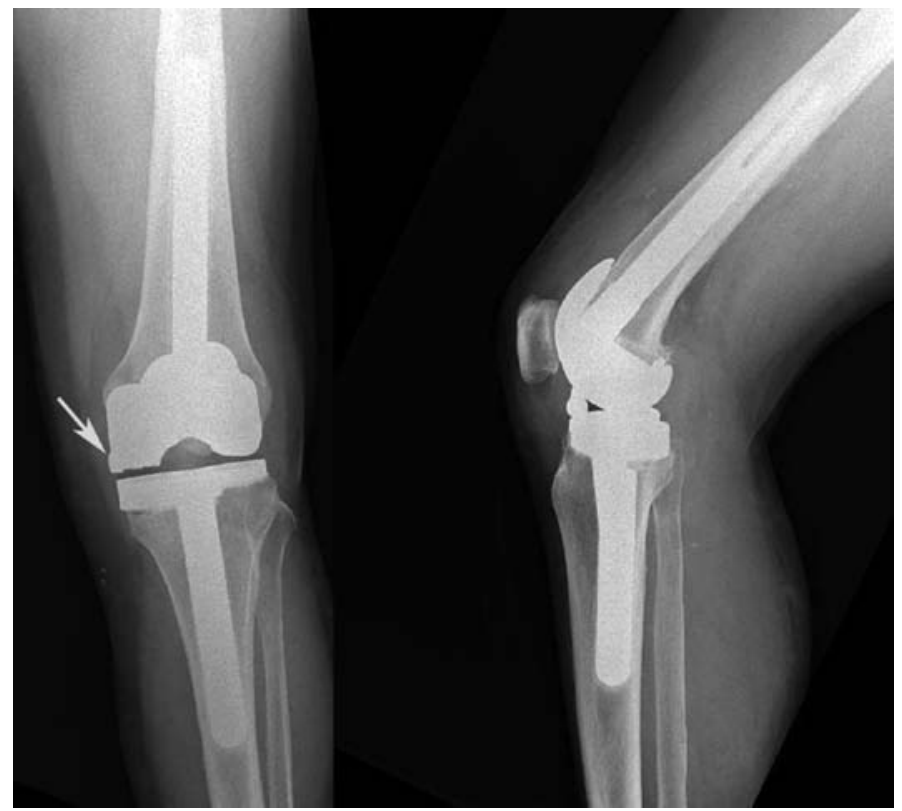

Figura 1 - Radiografia mostrando o parafuso no interior do joelho

Intra-operatóriamente verificou-se que o parafuso estava na goteira parapatelar medial e que a superfície articular femoral estava íntegra e sem danos (Figura 2). Não havia qualquer sinal de descolamento. Alterou-se a estratégia e procedeu-se à substituição do polietileno e à colocação de novo parafuso de bloqueio (Figuras 3 e 4).

A paciente teve um pós-operatório normal, sem incidentes, saindo do hospital ao $5 .^{\circ}$ dia após a cirurgia. A reabilitação incluiu o treino de amplitude articular.

Não se registaram mais problemas, tendo a paciente marcha normal e amplitude articular semelhante à do pré-operatório.

\section{DISCUSSÃO}

Tanto quanto é do nosso conhecimento, são raros os relatos na literatura de migração intra-articular de parafuso em $\operatorname{ATJ}^{(4,6,7)}$, havendo apenas um relato semelhante em artroplastia do tipo Performance, mas sem hastes tibiais e femorais ${ }^{(6)}$. Nesse tipo de artroplastias são escassos os relatos dos resultados a médio e longo prazo, referindo Basset ${ }^{(1)}$ que em 1.000 artroplastias desse tipo não observou qualquer complicação com o parafuso de bloqueio do polietileno.

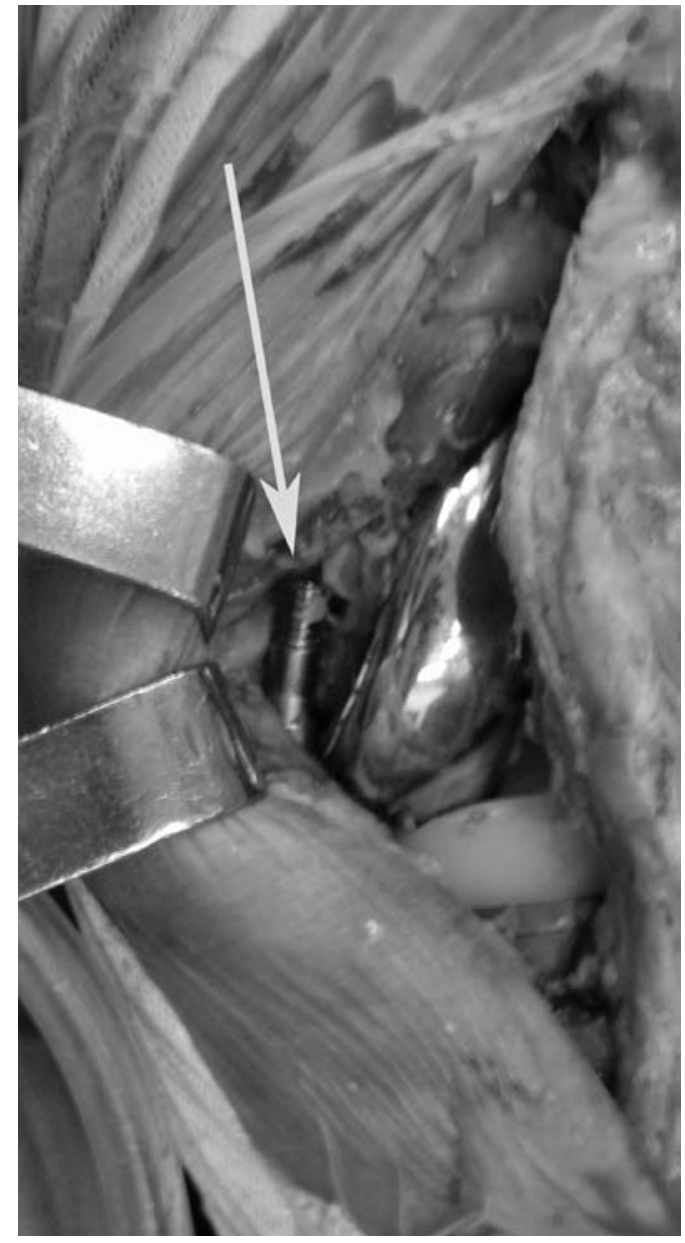

Figura 2 - Parafuso migrado

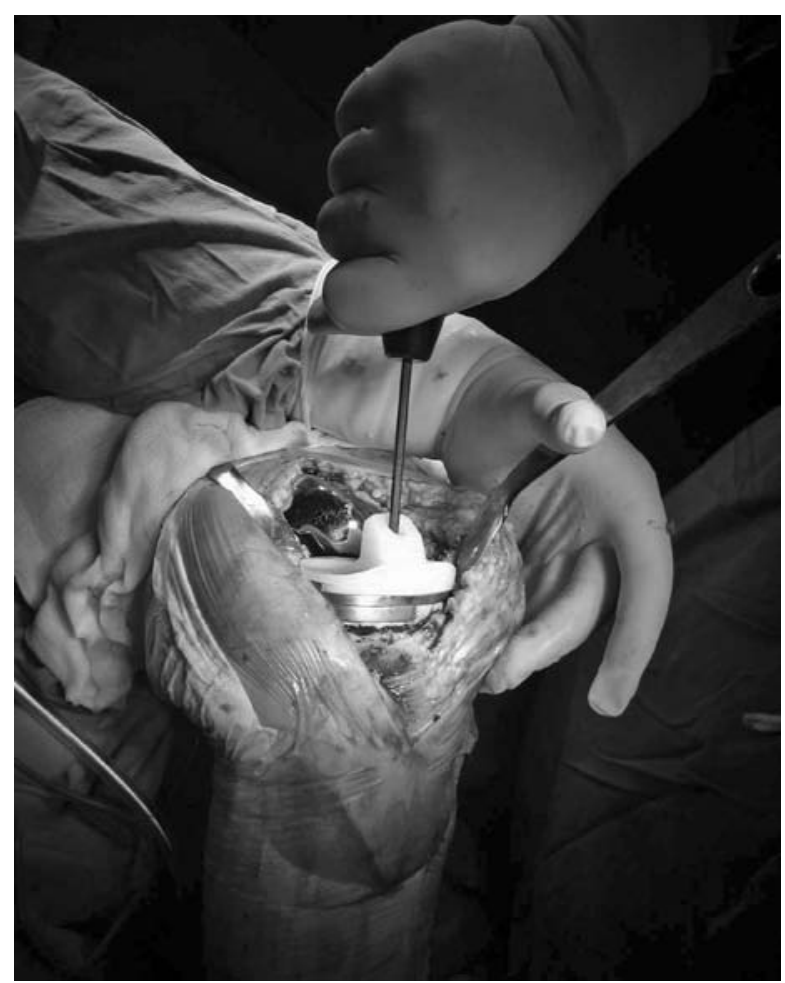

Figura 3 - Colocação de novo polietileno 


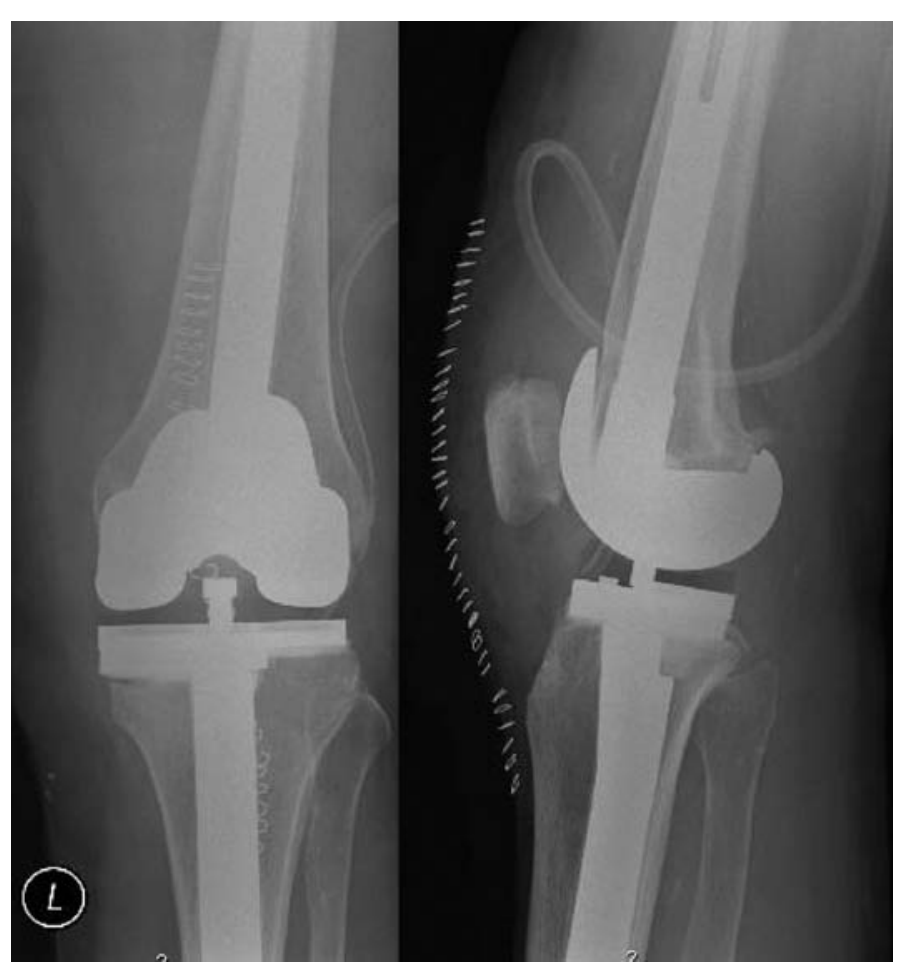

Figura 4 - Controlo pós-operatório
Ao contrário de outros relatos de migração de parafuso $^{(6)}$, este caso mostra a presença intra-articular de um parafuso actuando como um corpo livre, situação que não foi objecto de relato anterior.

A causa da migração ainda é pouco clara pois, como se pode ver na Figura 3, o parafuso fica dentro do polietileno. No registo da cirurgia inicial não houve qualquer relato de incidente e o parafuso foi colocado sob tensão de acordo com as normas definidas pelo fabricante.

O diagnóstico foi feito pelo controlo radiográfico, sendo a avaliação clínica pouco valorizada até à observação da radiografia.

Não temos a certeza se essa foi a melhor solução, mas os controlos nos dois anos seguintes têm mostrado que o parafuso está estável. Não temos outro caso de migração de parafuso como corpo livre.

Este caso alterou a nossa filosofia e determinou que se abandonasse a utilização de ATJs cujo polietileno seja fixado por parafuso de bloqueio.

Recomendamos que em artroplastia com esse sistema seja efectuado um controlo radiográfico frequente.

\section{REFERÊNCIAS}

1. Basset RW. Results of 1,000 Performance knees: cementless versus cemented fixation. J Arthroplasty. 1998;13(4):409-13.

2. Bayley JC, Scott RD, Ewald FC, Holmes GB Jr. Failure of the metal-backed patellar component after total knee replacement. J Bone Joint Surg Am. 1988;70(5):668-74.

3. Cameron HU, Welsh RP. Fracture of the femoral component in unicompartmental total knee arthroplasty. J Arthroplasty. 1990;5:(4):315-7.

4. Gradisar IA Jr, Hoffmann M, Askew MJ. Fracture of a fenestrated metal backing of a tibial knee component: a case report. J Arthroplasty. 1989;4(1):27-30.
5. Lachiewicz PF, Falatyn SP. Clinical and radiographic results of the total condylar III and constrained condylar total knee arthroplasty. J Arthroplasty. 1996;11(18):916-22.

6. Shah SN, Schurman DJ, Goodman SB. Screw migration from total knee prostheses requiring subsequent surgery. J Arthroplasty. 2002;17(7):951-4.

7. Westrich GH, Hidaka C, Windsor RE. Disengagement of a locking screw from a modular stem in revision total knee arthroplasty: a report of three cases. J Bone Joint Surg Am. 1997;79(2):254-8. 